\title{
DISTRIBUTED MODEL MANAGEMENT PLATFORM FOR VIRTUAL ENTERPRISE NETWORKS
}

\author{
Vanderhaeghen, Dominik; Loos, Peter \\ Institute for Information Systems (IWi) at the \\ German Research Center for Artificial Intelligence (DFKI) \\ Stuhlsatzenhausweg 3, Build. D3 2 \\ D-66123 Saarbrücken \\ GERMANY \\ \{dominik.vanderhaeghen|peter.loos\}@iwi.dfki.de
}

\begin{abstract}
Virtual enterprise network planning and design lead to complex challenges. From a conceptual point of view, business processes have proven to be the ideal design items in conjunction with the use of graphical methods and tools. Especially for network design and implementation, information modelling serves as the fundamental starting point. In the article, the authors depict a modelling approach dedicated to a global and a local description of business processes and a distributed model management platform. The latter supports the collaborative creation and application of models in network environments.
\end{abstract}

\section{INTRODUCTION}

Virtual enterprise (VE) network planning and design lead to complex challenges. Enterprise networks consist of autonomous economic entities, usually with a common target definition (Leimeister, Weigle, \& Krcmar, 2004). Additionally, due to numerous points of contact VEs are characterized by common resources and interfaces (Specht \& Kahmann, 2000). In a VE, at least two different organizations collaborate in order to create a common added-value. To do so efficiently, an integration and collaboration of network participants becomes necessary.

From a conceptual point of view, business processes have proven to be ideal design items in conjunction with the use of graphical methods and tools (Hammer \& Champy, 1993). However, with information gaps across the borders of an enterprise business process coordination turns out to be a highly complex task in the network. ${ }^{1}$ Thus, adequate instruments have to be designed and supported by Information and Communication Technology (ICT). For the planning and design of a VE network, information modelling serves as the fundamental starting point. A model depicts attributes and parameters necessary for requirement definitions. They comprise knowledge about business logic, data interfaces, or organizational responsibilities within a network. The structured design and provision of information for business and IT specialists is essential for an efficient setting-up of a network. As traditional

\footnotetext{
${ }^{1}$ Cf. requirements e.g. described in (Zang, Hofer, \& Adam, 2004).
} 
Business Process Management (BPM) approaches and tools do not provide support sufficiently, existing approaches and tools have to be extended.

The article depicts an approach towards general information modelling and model management in networks. The authors distinguish two modelling levels due to abstraction and selection of process information and the need for information security in cross-organizational collaborations. To support modelling tasks efficiently, an integrative platform for modelling and model management in distributed environments is described. Due to requirement descriptions have already been depicted in e.g. (Adam et al., 2005a) and (Theling et al., 2005) this article focuses on the description of solutions disregarding general requirements already described in former work. The article ends with a conclusion and an outlook on future research in the cross-organizational modelling and model management area.

\section{BUSINESS PROCESS MODELLING IN NETWORKS}

The systematic planning and design of business processes in VE networks demands a set of integrated methods and tools from the business concept level up to the implementation into ICT-systems. Existing BPM methods and phase models have to be used as a foundation and have to be adapted to the specifications of VE networks. Especially because of its completeness of vision and its proven practicability, both in the scientific and the economic context, the "ARIS House" (Scheer, 1994) is accepted as a generic framework for BPM. Hence, it serves as a basis for further considerations. The ARIS House describes a business process, assigning equal importance to the questions of organisation, functionality and the required documentation. First, it isolates these views for separate treatment in order to reduce the complexity of the description field, but then all the relationships are restored using a control view introduced for this purpose. Within the different ARIS views, information modelling serves as the main instrument.

To meet the design and implementation requirements of networks, instruments to describe as-is states and to design to-be states are considered in the dimensions of the ARIS House. Business analysts and IT specialists have to consider product and service descriptions, business process models and organisational structures for network planning, design and implementation. The transition between as-is and tobe in collaborative product development or process engineering is achieved by a successive synchronisation of organizations. Planning, design and implementation of a network require increased communication efforts between business partners involved. Thus, information is exchanged in the form of models which depict e.g. partner input/output descriptions, interface requirements, or process information.

Due to the characteristics of networks, knowledge is a critical resource. It is only shared with partners under given circumstances. Thus, based on (Adam, Chikova, Hofer, Zang, \& Vanderhaeghen, 2005a), knowledge is classified

- to be managed on a global level and such

- to be managed on a local level.

Both description levels form a knowledge classification which is represented in the ARIS House by a vertical axis (global knowledge) and a horizontal axis (local knowledge) (see Figure 1). 


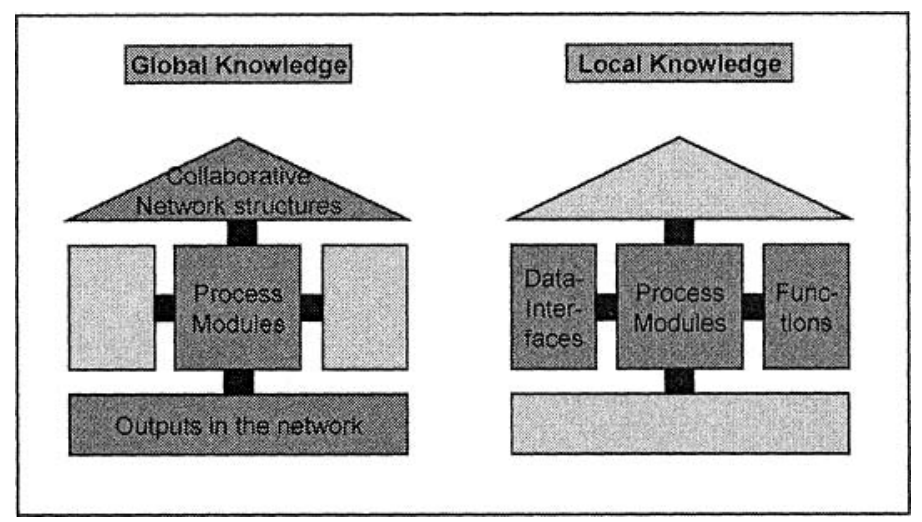

Figure 1 - Global and local knowledge in networks

Global knowledge comprises partner-spanning relevance on the one hand as e.g. a change of network structure due to partners leaving a network. This implies that products or services may be no longer available within the network. Thus, such information is globally important. Global models contain general conditions for e.g. application of ICT in a network. On the other hand, local knowledge as detailed processes or process interfaces is kept within the borders of an enterprise or bilaterally exchanged with direct business partners only. Consequently, models are required on a global and a local description and application level. While local information models are not made public to all business partners, global models intend a coordination of all partners.

To support local and global modelling in networks efficiently, the blueprint of a distributed model management platform is described in the following.

\section{DISTRIBUTED MODEL MANAGEMENT PLATFORM}

Networking enterprises implicates a close connection between discrete economic entities. However, the general design and implementation of internal business processes and ICT are usually managed without external exertion of influence. But at the same time internal organizational knowledge is shared selectively within enterprise networks in the form of models (Adam, Chikova, Hofer, Zang, \& Vanderhaeghen, 2005a). The intended coordination of business partners requires information processes between decision makers and actors in order to harmonize network actions. Thus, we propose an approach towards a distributed model management platform. To plan, to design and to implement networked business processes, modelling is conducted in a distributed manner between two or more organizations. Integration of models becomes a critical task due to heterogeneous modelling frameworks and modelling tools being in use in a network (Theling, Zwicker, Loos, \& Vanderhaeghen, 2005).

The authors describe a platform which is installed and applied in a VE network by every single participant organization. The platform mainly consists of a webbased front end, a model and model meta-data repository, interfaces to existing BPM 
tools and converters for the transfer and transformation of information models towards different syntactical representations. It is applied by multiple system instances which are able to communicate to each other. Main target is the management of local and global models due to the approach described in section 2. In the following, the authors revert to main challenges of the platform only. External integration of tools is not described.

\subsection{User Authorization and User Interface}

In the platform, a web-based user interface is provided to enable easy user access to information based on four different user roles (view user, modelling user, enhanced modelling user and administrator). Security requirements are met by a session-based module to manage login data. On a cross-organisational level, authentication is provided to external users by giving access on models which are stored in a single platform instance (c.f. also section 3.2). Single Sign-On (SSO) facilitates the work with different integrated modelling tools. The user interface provides a distinct navigation structure with multiple user information sets. Easy and quick access to basic functions is ensured by two navigation bars and links depending on the user role. Figure 2 illustrates the user interface with its basic functions (white boxes) and information sets (grey boxes).

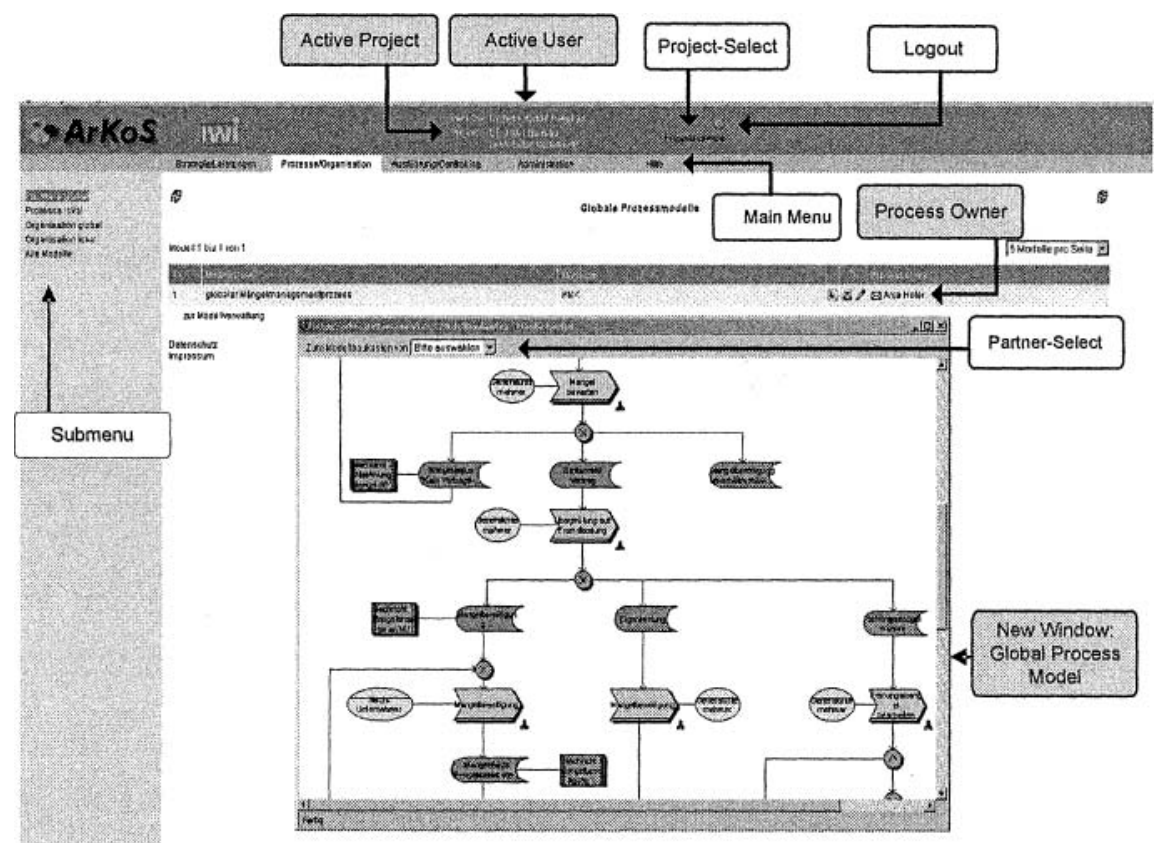

Figure 2 - Model Management User Interface 


\subsection{Information Access across multiple Platform Instances}

Network participants are allocated in distributed environments. The coordination of collaboration partners requires communication efforts. In our approach, this communication is realized with a distributed information exchange of models. However, in a network the exchange of information requires measures building trust between business partners (Ratnasingam, 2003).

On a communication layer, necessary access authorization for model exchange can be designed on a global level (ensuring communication between all partners) and a local level (Adam et al., 2005b). On a local level, communication is negotiated bilaterally (e.g. for information exchange between exact two partners). Based on these two levels of spatial distribution, access authorities are realized in two steps.

Firstly, unique access information is given to all partners in a network (global partner user name and global partner password). Access information is associated to one running project. The project is named unique by every partner. Conciliated access information is achieved in combination with a first network exchange of global models. Information models depicting the overall organizational network structure are transmitted to all partners for network management purposes. Thereby, partners get information in two different dimensions: On the one hand global information for network planning and design is exchanged with organisational models. These models also contain knowledge about project partners and mutual business process dependencies. Partner addresses (Unified Resource Locators (URL) of corresponding platform instances) are added as attributes to global organisational models. On the other hand, the global access data is inserted to the global model. On a protocol layer information access is encrypted via Internet by Secure Hyper Text Transfer Protocol (HTTPS).

Thereby, every partner gets all necessary information for a bilateral exchange of data between direct business partners. With

- the knowledge which partner combinations require bilateral communication in the network,

- single partner addresses (URL) known to the network,

- global and unique access information known to the network and

- secure communication channels with encryption mechanisms

the first stage of cross-organisational access framework is achieved. It enables data exchange in distributed environments.

With global knowledge about business partners, detailed information can be transferred in a second step. Partners once identify the need for communication within a network project by organisational dependency descriptions (cf. step 1). Consequently, business processes of business partners have cross-organisational interfaces to be aligned. Synchronisation and communication are facilitated by models and their bilateral exchange. Models depict necessary information on a partner's public processes and interface descriptions. They offer a possibility to recognize mutual dependencies and to react by process reengineering or customization of application systems. To facilitate the exchange of detailed models on a local (bilateral) level, every partner requests individual access information (partner's local user name and password) to its direct business partner. Hereby, the unique project identification number and globally known username and password are transmitted in association to the request. Partners check if a request is valid 
(correct partner request URL, correct project ID, correct global access information) and accept or deny a request. If accepted, a partner creates access information for the business partner request. If it is not valid, the request is ignored. Successful access is valid for a whole project duration and must not exceed a given time limit. With the created local user name and password, business partners get access to the information another partner has directly made public to him. This information is managed in platform instances in the form of models as public business process models, public ICT interface descriptions or product and service trees.

A cross-organisational and distributed communication between business partners is accomplished.

\subsection{Repository Storage and Distributed Information Exchange}

In a network, models might occur in different syntactical representations. This is related to the use of heterogeneous modelling languages and supporting tools. Control instances might be missing as some forms of collaboration networks only imply equal participants being part of a network. Examples are VE networks without one or more central, steering management occurrences. Thus, it is not possible to enforce a common syntactical representation for models or the use of special languages and tools. However, an agreement has to be established either on a local level between collaboration partners or on a global level to get a collaboration-wide and standardized default. In the latter case, the default needs a partner-wide acceptance. In the first case, an agreement between at least two network partners is satisfactory.

Coordination efforts in modelling need to be reduced or even minimized. A standardized description of information with one storage format becomes necessary. The format needs independency from tool providers as it requires a common acceptance among all network partners. The Business Process Modeling Notation (BPMN) provides an appropriate modelling language. It helps to describe process information on a conceptual level. The BPMN addresses modelling experts as well as IT specialists (White, 2004). It is supported by various tool providers. However, a standardized BPMN XML description format does not yet exist. Thus, based on the BPMN meta-model, a XML-based description and storage format has been designed and implemented as well as first JAVA transformation converters based on respective meta model - mappings between different methodologies (Theling, Zwicker, Loos, \& Vanderhaeghen, 2005; Vanderhaeghen, Zang, \& Scheer, 2005). Hereby, local process models in heterogeneous representations are transformed to BPMN-compliant representations and syntactically stored as BPMN XML models in a platform repository. With transformation and standardized representations, process models become syntactically understandable for all network participants. BPMN acts as a common denominator for information description and information storage.

\subsection{Model Synchronisation}

To apply existing models of participants in a VE network, private models from BPM or software engineering are selected due to their relevance for network coordination. Hereby, a selection of models is copied and stored in the model management platform with a unique backtracking to their original model instances. The assumed 
repository of private process models are hidden to business partners and managed in separate tools not accessible to unauthorized, external people. Thereby, critical information models which must not be made public are protected. Participants of a network are able to manage their collaborative relationships with a pool of selected models being necessary for network and partner coordination.

Figure 3 depicts the proposed synchronization procedure which assures that model copies - selected for collaborative application and thus managed in the platform - enable retraceability to their local source pendants. A need for model alignment is identified by working with these copies of models. With new collaborative requirements, private models may be changed and updated for their collaborative application afterwards.

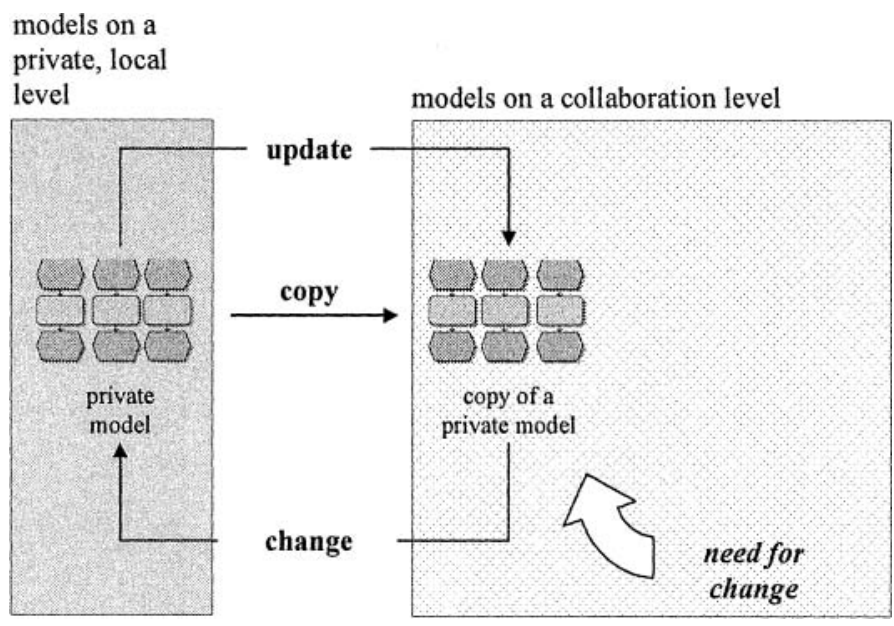

Figure 3 - Synchronization Procedure

\section{CONCLUSIONS AND FUTURE WORK}

With a vast variety of influencing factors, VE partner coordination becomes a highly complex task. This complexity needs to be reduced by appropriate methods and tools. The need for special coordination efforts and the development of enhanced methods and tools for modelling in networks has been derived from former research results. Information modelling could be identified as a well-established instrument for the design and implementation of enterprises and their role in VE networks. However, new requirements towards an enterprise-spanning modelling have to be considered due to existing approaches do not support modelling in VEs sufficiently.

The authors have presented an integrative approach towards the management of cross-organisational models in networks. Efficient modelling is supported by a platform for a distributed model management which has been partly described due to VE network requirements in this article. However, the platform approach has to be evaluated in future work. Hereby, case studies are currently being developed to be carried out in practice. New requirements towards information modelling in VE 
networks might arise which have to be taken into account for network model management.

The work, described in this paper, has been developed within the research project "ArKoS - Architecture for Collaborative Scenarios". The project is funded by the German Federal Ministry of Education and Research (BMBF). The authors want to thank Mr. Adam, Mrs. Hofer and Mr. Zang as well as Mr. Panfilenko and Mr. Woldert-Jokisz for their contribution within the project.

\section{REFERENCES}

1. Adam, O., Chikova, P., Hofer, A., Zang, S., Vanderhaeghen, D. "e-Collaboration architecture for customer-driven business processes in inter-organizational scenarios". In Challenges of Expanding Internet: E-Commerce, E-Business, And E-Government. M. Funabashi \& A. Grzech, eds., Berlin: Springer, 2005; 63-77.

2. Adam, O., Hofer, A., Zang, S., Hammer, C., Jerrentrup, M., Leinenbach, S. "A Collaboration Framework for Cross-enterprise Business Process Management". In Preproceedings of the First International Conference on Interoperability of Enterprise Software and Applications NTEROPESA'2005 Geneva, Switzerland, February 23 - 25, 2005; 499-510.

3. Hammer, M., Champy, J. Reengineering the corporation : a manifesto for business revolution. New York: Harper Business, 1993.

4. Leimeister, J. M., Weigle, J., Krcmar, H. Effizienz virtueller Unternehmen: Ein Vergleich zweier Projekte bei AGI. Hohenheim: Universität Hohenheim, 2004.

5. Ratnasingam, P. 2003, Inter-Organizational Trust for Business-to-Business E-commerce. Hershey et al.: IRM Press.

6. Scheer, A.-W. Business Process Engineering : Reference Models for Industrial Enterprises. Berlin: Springer, 1994.

7. Specht, D., Kahmann, J. "Regelung kooperativer Tatigkeit im virtuellen Unternehmen". In Virtuelle Unternehmen, H. Albach, D. Specht, H. Wildemann, Gabler, 2000; 55-73.

8. Theling, Th., Zwicker, J., Loos, P., Vanderhaeghen, D. "An Architecture for Collaborative Scenarios applying a common BPMN-Repository". In Kutvonen, L; Alonistioti, N.: Distributed Applications and Interoperable Systems: 5th IFIP WG 6.1 International Conference, DAIS 2005, Athens, Greece, June 15-17, 2005. Lecture Notes in Computer Science, Volume 3543, Berlin: Springer-Verlag; $169-180$.

9. Vanderhaeghen, D., Zang, S., Scheer, A.-W. Interorganisationales Geschaftsprozessmanagement durch Modelltransformation. Saarbrücken: Universităt des Saarlandes, Saarbrücken, 2005; 182.

10. White, S. A. Business Process Modeling Notation (BPMN). BPMI.org. http://www.bpmi.org/bpmi-downloads/BPMN-V1.0.pdf . 2004.

11. Zang, S., Hofer, A., Adam, O. "Cross-Enterprise Business Process Management Architecture Methods and Tools for Flexible Collaboration" In On the Move to Meaningful Internet Systems 2004: OTM 2004 Workshops, R. Meersmann, Z. Tari, \& A. Corsaro, eds., Berlin: Springer Verlag, Berlin, 2004; 483-494. 